\section{Metastatic plasma cell leukemia to the skin: A case report with review of the literature}

\author{
Elena Pezzolo,,${ }^{1-3}$ Deborah Saraggi, ${ }^{4}$ \\ Luigi Naldi ${ }^{1,3}$ \\ ${ }^{1}$ Department of Dermatology, San \\ Bortolo Hospital, Vicenza, Italy; \\ ${ }^{2}$ Department of Epidemiology, Erasmus \\ MC University of Rotterdam, The \\ Netherlands; ${ }^{3}$ Study Center of the \\ Italian Group for Epidemiologic \\ Research in Dermatology (GISED), \\ Bergamo, Italy; ${ }^{4}$ Department of \\ Pathology, San Bortolo Hospital, \\ Vicenza, Italy
}

\begin{abstract}
Plasma cell leukemia (PCL) is a rare variant of leukemia with an aggressive clinical course and a poor prognosis. The cutaneous involvement in PCL is very rare either at clinical presentation of leukemia, namely leukemia cutis, or in the metastatic PCL to the skin. We present a case of eruptive multiple cutaneous nodules in a 56-year-old man with metastatic PCL. Histologically, a diffuse dermal and subcutaneous infiltration of ovoid cells with amphophilic cytoplasm and eccentrically located nucleus consistent with plasmacytoid morphology was observed. Neoplastic cells showed strong immunoexpression for CD138 and CD38 consistent with plasma cells phenotype, and loss of expression of CD56. Kappa light chain restriction similar to the phenotype of his PCL was demonstrated. We suggest that the evaluation of new skin lesions in leukemic patients should include a histopathologic examination to establish the diagnosis as soon as possible and a correct management of the disease.
\end{abstract}

\section{Introduction}

Plasma cell leukemia (PCL) is an aggressive, very rare variant of leukemia that represents only two percent of plasma cell neoplasms. ${ }^{1}$ The disease is defined as a clonal proliferation of plasma cells in the blood and the bone marrow, in which the absolute plasma cells count is greater than $2 \times 10^{9} / \mathrm{L}$ in a peripheral blood smear, or plasma cells comprise greater than $20 \%$ of the total peripheral leukocytes. ${ }^{2}$ There are two forms of this lymphoproliferative disorder: primary PCL is defined as a malignant plasma cell proliferation first diagnosed in the leukemic phase (the form which occurs de novo, in the absence of multiple myeloma), while secondary PCL corresponds to the leukemic transformation of a previously diagnosed multiple myeloma. Both the primary and the secondary forms of PCL share an aggressive clinical course and a poor prognosis. The clinical manifestations may include extramedullary plasma cell infiltration, mainly of liver and spleen, but also infiltration of other visceral sites such as the kidneys, lungs, heart, pleura, testes, skeletal muscles, and central nervous system.

The cutaneous involvement in PCL, indicating an infiltration of the skin by malignant leukocytes or their precursors, is very rare either at clinical presentation of leukemia, namely leukemia cutis, or in the metastatic PCL to the skin. We here present a case of eruptive multiple cutaneous nodules in primary PCL and the results of a literature review.

\section{Case report}

A 56-year-old man presented to the dermatology clinic with a 15-day history of painless brownish nodular lesion on his right side. He had a 1-year history of primary PCL type kappa with circulating plasma cells corresponding to $24 \%$ of leukocytes, for which he had been candidate to receive autologous stem cell transplant. Physical examination revealed a large erythematous, firm, non-tender and smooth nodule with hemorrhagic base and small ecchymotic foci on the surface, extending for $3 \times 2 \mathrm{~cm}$ in diameter on the trunk (Figure 1). There was no significant lymphadenopathy.

Complete blood count revealed hemoglobin $12.8 \mathrm{~g} / \mathrm{dL}$ (normal: 12.4-14.9 $\mathrm{g} / \mathrm{dL}$ ), red blood cells $3.9 \times 10^{12} / \mathrm{L}$ (normal: $\left.4.53-5.90 \times 10^{12} / \mathrm{L}\right)$, white blood cells $4.5 \times 10^{9} / \mathrm{L}$ (normal: $4-11 \times 10^{9} / \mathrm{L}$ ) with $64.6 \%$ neutrophils (normal: $40-75 \%$ ), and platelets $148 \times 10^{9} / \mathrm{L}$ (normal: $150-400 \times 10^{9} / \mathrm{L}$ ).

Biochemical parameters were serum creatinine $0.8 \mathrm{mg} / \mathrm{dL}$ (normal: 0.8-1.3 $\mathrm{mg} / \mathrm{dL}$ ), serum calcium $9.5 \mathrm{mg} / \mathrm{dL}$ (normal: 8.9-10.1 $\mathrm{mg} / \mathrm{dL}$ ), total serum proteins 7.2 g/dL (normal: 6.4-8.3 g/dL), lactate dehydrogenase (LDH) 187 U/L (normal: 122$222 \mathrm{U} / \mathrm{L}$ ), and $\mathrm{b}_{2}$ microglobulin $2.1 \mathrm{mg} / \mathrm{dL}$ (normal: $<0.2 \mathrm{mg} / \mathrm{dL}$ ). His serum bilirubin and liver enzymes were within normal limits. Serum protein electrophoresis revealed monoclonal gammopathy. Serum free kappa light chain was increased to $33.7 \mathrm{mg} / \mathrm{L}$ (normal: 3.3-19.4 mg/L) with kappa: lambda ratio of $12.4: 1$. Bacterial, fungal and mycobacterial cultures were obtained, to exclude infectious causes in the differential diagnosis of the nodule. An incisional biop-
Correspondence: Elena Pezzolo, Department of Dermatology, San Bortolo Hospital, Viale Ferdinando Rodolfi, 37, 36100 Vicenza (VI), Italy.

E-mail: elena.pezzolo@gmail.com

Key words: Leukemia cutis; Plasma cell leukemia; Nodules; Histopatology.

Contributions: Conceptualization: EP, DS. Data curation: EP, DS. Investigation: EP, DS Methodology: EP, DS. Project administration: EP. Resources: EP, DS. Supervision: LN. Visualization: EP, DS, LN. Writing (original draft): EP, DS. Writing (review and editing): EP, DS, LN. EP and DS contributed equally.

Conflict of interest: The authors declare no potential conflict of interest.

Funding: None.

Ethical approval: This study was approved by the local institution' ethics committee.

Consent for publication: Patient gave his informed consent for the publication of data.

Availability of data and material: Data and materials are available by the authors.

Please cite this article as: Pezzolo E, Saraggi $D$, Naldi L. Metastatic plasma cell leukemia to the skin: A case report with review of the literature. Dermatol Rep 2021;13:9099.

Received for publication: 11 February 2021. Revision received: 27 May 2021.

Accepted for publication: 20 June 2021.

This work is licensed under a Creative Commons Attribution-NonCommercial 4.0 International License (CC BY-NC 4.0).

(Copyright: the Author(s), 2021

Licensee PAGEPress, Italy

Dermatology Reports 2021; 13:9099

doi:10.4081/dr.2021.9099

sy for histopathologic examination was performed. The biopsy showed diffuse infiltration of ovoid cells with amphophilic cytoplasm and eccentrically located nucleus consistent with plasmacytoid morphology. The infiltrate involved the entire thickness of the dermis, and also extended to subcutaneous tissue while the epidermis was spared (Figures 2 and 3). Neoplastic cells showed strong immunoexpression for CD138, CD38, MUM1, CyclinD1 and loss of expression of CD56. Kappa light chain restriction similar to the phenotype of his PCL was demonstrated.

A diagnosis of cutaneous metastatic PCL was made. A Positron Emission Tomography (PET) scan showed extra-medullary disease 
sites involvement at bones, lungs, and liver. Similar but smaller subcutaneous nodules developed 15 days thereafter on his lower back, which were surgically excised. Patient's disease course had been progressive, despite treatment with bortezomib-based chemotherapy regimens plus the immunomodulatory drug thalidomide: the patient ultimately died 3 months later due to lung infection and sepsis.

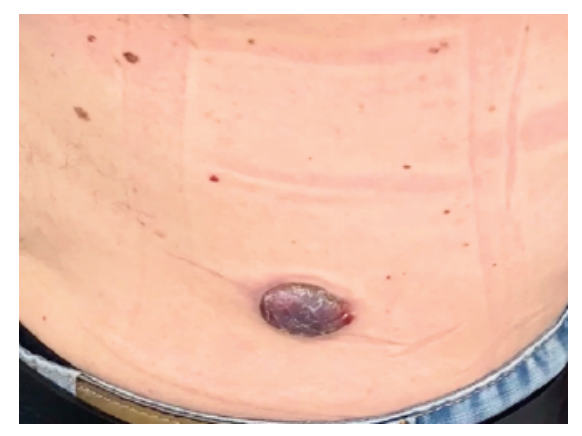

Figure 1. A large erythematous, non-tender nodule with a hemorrhagic base, violaceus margins and small ecchimotic foci on the surface, extending for $3 \times 2$ centimeters in diameter on the trunk.

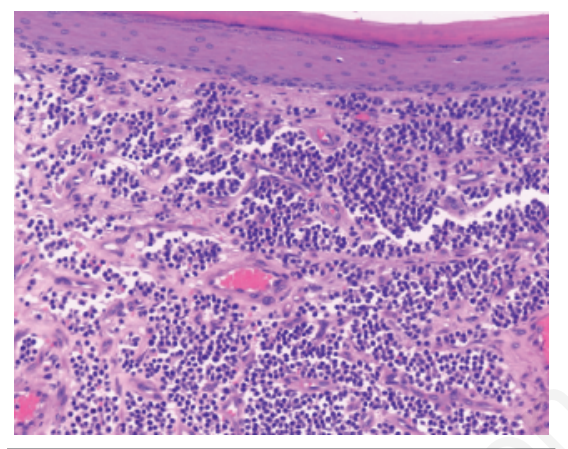

Figure 2. Hematoxylin and eosin stain (20x magnification). Scanning magnification demonstrated diffuse dermal infiltration of neoplastic cells. The epidermis is not involved.

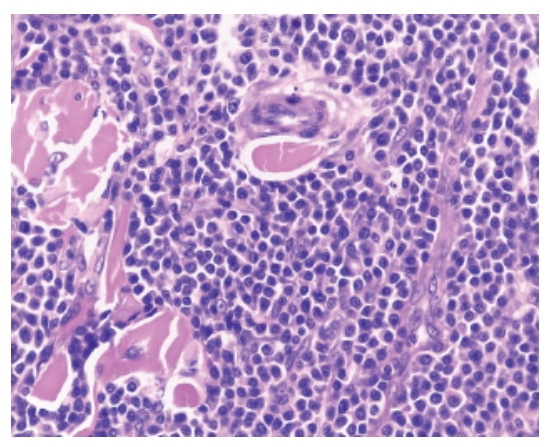

Figure 3. Hematoxylin and eosin stain (40× magnification). Higher magnification showed neoplastic cells with plasmacytoid cytomorphology.

\section{Discussion and conclusions}

We reported a rare case of cutaneous infiltration by neoplastic cells in primary PCL. Extramedullary involvement is known to occur in primary PCL, though cutaneous infiltration by malignant plasma cells at presentation of PCL or at its metastatic phase is a rare event: in the former the disease is named leukemia cutis, while in the latter it is named metastatic PCL. Due to the low frequency of this entity, most publications on primary PCL are based on case reports. Eruptive metastatic nodules to the skin in primary PCL was first described by Osanto et al. in $1983,{ }^{3}$ and, as far as we know, very few additional cases have been reported since, consisting mainly of middle-aged patients who developed multiple subcutaneous nodules after complete clinical remission achieved through chemotherapy. ${ }^{3-5}$ Moreover, the review of the literature regarding specific cutaneous involvement in primary PCL is difficult to interpret, as some cases were reported as part of series where individual details were missed. In a case series of 26 patients with primary PCL, only three cases among those presenting extramedullary involvement had subcutaneous nodules. ${ }^{6}$ Additional four cases of primary PCL manifesting nodular skin infiltration resembling urticaria, ${ }^{7}$ or painful nodular lesions over arms, trunk and buttocks, ${ }^{8}$ or erythematous nodules on lower limbs, abdomen and shoulders were reported in literature as unusual PCL presentation, ${ }^{9}$ as cutaneous involvement occurred at presentation of leukemia, and they were cases of leukemia cutis. The clinical lesions observed in our patient are similar to the cutaneous nodules described in a 62-year-old man with secondary PCL: erythematous, non-tender lesions with violaceus margins and small ecchimotic foci in the surface..$^{10}$ Other clinical data observed in our patient are in line with previous reports showing that primary PCL patients usually have extramedullary disease and anemia. ${ }^{3-5}$ However, our case did not had thrombocytopenia, hypercalcemia, impaired renal function, neither increased LDH or $b_{2}$ microglobulin, which are usually associated with aggressive course.

Histologically, in all cases reported it was noted a diffuse dermal or subcutaneous infiltration by neoplastic cells. Immunohistochemical studies in all cases demonstrated strong immunoexpression for CD138 and CD38 consistent with plasma cells phenotype. In contrast with other plasma cell neoplasms, PCL shows less frequent expression of CD56, which is negative in about $80 \%$ of PCLs, as in our case. ${ }^{11}$ As expected, in each literature case the light chain type expression in the skin correlated with bone marrow. In contrast with normal plasma cells, neoplastic cells of PCL may show aberrant expression of CD56, as in our case. In conclusion, cutaneous involvement by plasma cell leukemia occurs very rarely and heralds a very poor prognosis. The evaluation of new skin lesions in leukemic patients should include a histopathologic examination to establish the diagnosis as soon as possible and a correct management of the disease.

\section{References}

1. Mina R, D'Agostino M, Cerrato C, et al. Plasma cell leukemia: update on biology and therapy. Leuk Lymphoma 2017;58:1538-47.

2. Kyle RA, Maldonado JE, Bayrd ED. Plasma cell leukemia: report of 17 cases. Archs Intern Med 1974;133:813-8.

3. Osanto S, Müller HP, Schuit HRE, et al. Primary plasma cell leukemia - a case report and a review of the literature. Acta Haemat 1983;70:122-9.

4. Bareford D, Pamphilon DH, Barnard DL. Plasma cell leukemia relapsing in the dermis. Acta Haemat 1984;71:359.

5. Suzuki M, Kawauchi K, Sugiyama H, et al. Primary plasma cell leukemia: a case report of successful responder to a combination chemotherapy of vincristine, doxorubicin and dexamethasone. Acta Haemat 1989;82:95-7.

6. García-Sanz R, Orfão A, Gonzáles M, et al. Primary plasma cell leukemia: clinical, immunophenotypic, DNA ploidy, and cytogenetic characteristics. Blood 1999;93:1032-7.

7. Robak T, Urbanska-Ryś H, Bartkowiak J, et al. Aggressive primary plasma cell leukemia with skin manifestations, trisomy 8 and molecular oligoclonal features. Leuk Lymphoma 2002;43:1067-73.

8. Chattopadhyay A, Nath UK, De R, et al. Primary plasma cell leukemia with initial cutaneous involvement and IgA biclonal gammopathy. Ann Hematol 2008;87:249-51.

9. Gupta R, Nath AK, Subbian M, et al. Hemorragic skin nodules and plaques: a diagnostic clue to underlying primary plasma cell leukemia. Indian J Dermatol 2016;61:203-5.

10. De Martinis NC, Brown MM, Hinds BR, Cohen PR. Leukemia cutis associated with secondary plasma cell leukemia. Cureus 2017;9:e1235.

11. Fernandez de Larrea C, Kyle RA, Durie $\mathrm{BG}$, et al. Plasma cell leukemia: consensus statement on diagnostic requirements, response criteria and treatment recommendations by the International Myeloma Working Group. Leukemia 2013;27:780-91. 